\title{
Long-term suprapubic catheterisation: clinical outcome and satisfaction survey
}

\author{
MKM Sheriff, S Foley, J McFarlane, R Nauth-Misir, M Craggs and PJR Shah \\ Spinal Injuries Unit, Royal National Orthopaedic Hospital, Stanmore and Institute of Urology and Nephrology, \\ St Peter's Hospital, London, UK
}

\begin{abstract}
We report on the clinical outcome and satisfaction survey of long-term suprapubic catheterisation in patients with neuropathic bladder dysfunction. Between early 1988 and later 1995, 185 suprapubic catheters were inserted under direct cystoscopic vision. Anticholinergic therapy was given to all patients with significant detrusor hyper-reflexia; the catheters clamped daily for two hours and changed every six weeks. Ultrasonography and assessment of the serum creatinine were used to assess the upper renal tracts, and the results of the pre-and post-catheter video-cystometrography was used to evaluate bladder morphology, cystometric capacity, maximum detrusor pressure and the presence of vesico-ureteric reflux. There were equivalent numbers of males and females. The follow-up ranges from 3-68 months. Following catheterisation, there was a $50 \%$ reduction in the average maximum detrusor pressure, bladder morphology improved in $85 \%$ of the cases; the bladder capacity and upper renal tracts remained unchanged. Vesico-ureteric reflux was abolished in $33 \%$ of the cases. Complaints were common consisting of recurrent catheter blockage, persistent urinary leakage and recurrent urinary tract infections. There was a $2.7 \%$ incidence of small bowel injury with one fatality. However, the general level of satisfaction was high. It is concluded that suprapubic catheterisation is an effective and well tolerated method of management in selected patients with neuropathic bladder dysfunction for whom only major surgery would otherwise provide a solution to incontinence. We are encouraged to find preservation of renal function with maintained bladder volumes and reduced maximum detrusor pressures thus justifying the policy of catheter clamping and anti-cholinergic therapy in the presence of significant detrusor hyper-reflexia. However, even in expert hands this procedure is not without hazards.
\end{abstract}

Keywords: suprapubic catheters; catheterisation; detrusor pressure; satisfaction; clinical outcome

\section{Introduction}

In 1976 Cook and Smith described the use of suprapubic catheterisation (SPC) for the acute or elderly management of the neuropathic bladder. ${ }^{1}$ Today, long-term urinary catheterisation remains a valuable option in the management of both neuropathic and non-neuropathic bladder dysfunction. However, this technique has met both enthusiasm and criticism. In our unit despite the policy of clean self-intermittent catheterisation (CSIC), we have accrued a group of patients who are managed by long-term suprapubic catheters on our outpatient basis. Suprapubic is preferred to urethral catheterisation as the latter is associated with urethral damage. ${ }^{2,3}$ This form of management has been successfully employed by several centres for the treatment of continued urinary incontinence in the female, ${ }^{4}$ but has been

Correspondence: MKM Sheriff reported to be associated with accelerated renal deterioration in the spinal injury group. ${ }^{5}$

This unit first introduced a policy of suprapubic catheter management consisting of anti-cholinergic therapy in the presence of significant detrusor hyperreflexia to suppress detrusor function, daily catheter clamping for $2 \mathrm{~h}$ to maintain the reservoir capacity of the bladder, and a catheter change every 6 weeks. ${ }^{6}$ By these means it was hoped to avoid the development of upper urinary tract abnormalities in the long-term. The demand for this convenient form of bladder management has continued to increase and therefore further assessment of its clinical implications and acceptance in long-term use is required. In this study we aim to answer three questions, first, what is the current place of long-term suprapubic catheterisation in the management of the neuropathic bladder, secondly how should these catheters be best managed and finally what do the patients think about this form of bladder management. 


\section{Materials and methods}

We have reviewed the records of 185 patients who have had SPC inserted between early 1988 and late 1995. This represents $23 \%$ of all new patients referred to our Neuro-urological Unit during this time. One hundred and fifty-seven complete records out of the total 185 were available for analysis. All SPC were inserted under cystoscopic control to ensure correct positioning of the catheter tip within the bladder, the optimal position being the dome of the bladder away from the bladder neck. This position avoids complications such as urinary retention following the placement of the catheter tip too close to the bladder neck. The 'ADDa-CATH' or the Nottingham type of catheter was used in the majority of cases, rarely the Hey-Groves and the Argyl were employed. Size 16F pure silicone catheter was used in over $90 \%$ of the cases. Patients prone to autonomic dysreflexia were given general anaesthesia in order to prevent complications. Anti-cholinergic therapy was given to patients with clinically significant detrusor hyper-reflexia, patients encouraged to clamp the catheters daily for $2 \mathrm{~h}$ and the catheters changed every 6 weeks. Renal function and morphology was assessed annually by estimation of serum creatinine and ultrasonography. Video-cystometrography (VCMG) was carried out before and following SPC by the same experienced individual with identical conditions as far as possible using Urografin ${ }^{\circledR}$ (Scherine AG) radiological contrast medium warmed to room temperature and at a slow-fill rate of $10 \mathrm{ml}$ per min. These results have been employed to assess the bladder morphology, cystometric bladder capacity, maximum detrusor pressure, and the presence of vesico-ureteric reflux. All patients or carers were requested to complete a satisfaction survey. The changes in cystometric bladder capacity and maximum detrusor pressure, were analyzed by using students $t$ test.

\section{Results}

One hundred and thirty-eight out of the 157 patients had traumatic neuropathic bladder dysfunction mostly as a result of cervico-thoracic spinal cord involvement and the remainder were predominantly patients with multiple sclerosis. There were 80 males and 77 females with an age range of 17 to 71 years, with a mean of 35 years amongst the males and 40 years amongst the females. Follow-up ranges from 3-68 months, with a mean of 24 months. There were eight patients with SPC for longer than 2 years. The main reason for SPC was failed CISC due to poor hand function (Table 1). However, in $20 \%$ of the cases it was to replace urethral catheters inserted elsewhere and interestingly in $18 \%$ of the cases the catheter was inserted by the request of the patient having heard about this form of bladder management from other spinally injured patients with the SPC. Whilst clearly one cannot always fulfil patient's wishes, however, in the absence of a better
Table 1 Indications for SPC

\begin{tabular}{lc}
\hline Indication & Number \\
\hline Failed CISC & 63 \\
$\quad$ Poor hand function (32) & \\
Persistent incontinence (15) & \\
Recurrent urinary tract infections (10) & \\
Bulbar strictures (6) & 22 \\
Replace urethral catheters & 18 \\
Patient request & 5 \\
Other & \\
\hline
\end{tabular}

Table 2 VCMG results before and after SPC

\begin{tabular}{lcc}
\hline & Pre-SPC & Post-SPC \\
\hline $\begin{array}{l}\text { Cystometric bladder } \\
\text { capacity (mls) }\end{array}$ & $367.4 \pm 57.3$ & $289.6 \pm 57.3$ \\
$\begin{array}{l}\text { Mean maximum } \\
\text { detrusor pressure } \\
\left(\mathrm{cms} / \mathrm{H}_{2} \mathrm{O}\right)\end{array}$ & $76.7 \pm 10.7$ & $(P=0.09)$ \\
$\begin{array}{l}\text { Vesico-ureteric } \\
\text { reflux }\end{array}$ & $38.4 \pm 5$ \\
$\begin{array}{l}\text { Cystometric bladder } \\
\text { morphology }\end{array}$ & trabeculated & $(P=0.002)$ \\
& & smooth in $85 \%$ \\
\hline
\end{tabular}

alternative or clinical contraindications, we believe that it is reasonable to insert a SPC. Complete VCMG results were available in twenty-one patients (Table 2). It should be noted that all of these 21 patients were on anti-cholinergic treatment throughout the study and there was no change in treatment between the initial and the follow-up VCMG. Assessment of cystometric bladder morphology is clearly subjective and rather imprecise, nevertheless, there was $85 \%$ improvement in bladder morphology as assessed by the same experienced radiologist. There was an apparent fall in the average cystometric bladder capactity from $367 \mathrm{ml}$ to $289 \mathrm{ml}$ after SPC. However, it is important to appreciate that this was not found to be statistically significant $(P=0.09)$ and therefore it is reasonable to state that the actual cystometric bladder capacity was maintained.

The average maximum detrusor pressure fell by $50 \%$ from $76.7 \mathrm{~cm} \mathrm{H}_{2} \mathrm{O}$ to $38.4 \mathrm{~cm} \mathrm{H}_{2} \mathrm{O}$ following SPC. This was found to be a statistically significant $(P=0.002)$ reduction. Grade IV Vesico-ureteric reflux was present in three out of the 21 cases before the insertion of the SPC. Following SPC, VCMG demonstrated persisted grade IV reflux in two patients and an absence of reflux in one patient. Although all three patients were on anticholinergic treatment throughout the study, interestingly the two patients with no change in vesico-ureteric reflux were on free bladder drainage unlike the third patient. However, there are several other factors which may have influenced this change, therefore this finding must be interpreted with due caution. 
Table 3 Common complaints following SPC

\begin{tabular}{|c|c|c|c|}
\hline & Clamp and suppressed & Clamp only & Neither \\
\hline Group incidence & $\begin{array}{c}25 \% \\
(n=27)\end{array}$ & $\begin{array}{c}32 \% \\
(n=36)\end{array}$ & $\begin{array}{c}38 \% \\
(n=42)\end{array}$ \\
\hline Blockage & $19 \%$ & $15 \%$ & $20 \%$ \\
\hline Recurrent UTI's & $6 \%$ & $5 \%$ & $6 \%$ \\
\hline Leakage & & $12 \%$ & $12 \%$ \\
\hline \multicolumn{4}{|c|}{$30 \%$ overall incidence } \\
\hline \multirow{3}{*}{\multicolumn{3}{|c|}{$\begin{array}{l}\text { - Blockage } \\
\text { - Recurrent symptomatic urinary tract infection (UTI's) } \\
\text { - Leakage }\end{array}$}} & $18 \%$ \\
\hline & & & $4 \%$ \\
\hline & & & $8 \%$ \\
\hline
\end{tabular}

Table 4 Specific complications of SPC and their management

\begin{tabular}{lc}
\hline Complication & Number \\
\hline Small bowel injury & 5 \\
- 3 resolved on conservative management & \\
- 1 died from silent peritonitis & \\
- 1 underwent inappropriate laporatomy & \\
Haemorrhage (settled with a & 2 \\
$\quad$ myocutaneous stitch) & 2 \\
Fail to drain (required catheter & \\
$\quad$ repositioning) & 1 \\
Fell out (required catheter re-insertion) & 8 \\
Persistent leakage: Managed as follows & \\
- Conservative (1) & \\
- Clam ileocystoplasty (1) & \\
- Ileal conduit (1) & \\
- Revision SPC (1) & \\
- Endoscopic bladder neck suspension (2) \\
- Urethrocleisis (1) \\
- Kaufman Procedure (1)
\end{tabular}

In all patients managed by SPC for more than 2 years, renal function as assessed by serum creatinine levels before and after insertion remained stable (mean before $=78 \mu \mathrm{ml} / 1$, after $=65 \mu \mathrm{ml} / 1)$. Ultrasonography showed no upper tract dilatation or demonstrable cortical thinning.

In our series $92 \%$ of the patients had their catheters changed between 6-8 weeks, with $83 \%$ at the recommended 6 weeks. Furthermore, the majority $(96 \%)$ of the patients practised catheter clamping (independently or by the carer), with $75 \%$ for the recommended $2 \mathrm{~h}$ daily. If we consider who actually changed the catheters, it may be surprising to note that the majorities were done by the hospital staff. The general practitioner contributed in only $8 \%$ of the cases, the district nurse in $21 \%$ and the patient or carer in $18 \%$ of the cases.

There was a significant $30 \%$ overall incidence of complaints. The commonest complaint was recurrent catheter blockage in $18 \%$ of the cases followed by persistent urinary leakage in $8 \%$ and recurrent symptomatic urinary tract infections in $4 \%$ (Table 3). However, the overall incidence of asymptomatic urinary tract infections was in the region of $98 \%$. Forty-eight percent of the patients $(n=52)$ with complaints had associated bladder calculi requiring intervention.

There was a $10 \%$ incidence of complications with a $2.7 \%$ incidence of small bowel injury (Table 4 ). Three of those with bowel injury resolved conservatively, one underwent inappropriate surgical intervention elsewhere, and the other patient who had a high complete spinal cord injury died from unrecognised silent peritonitis.

The satisfaction questionnaire was successfully completed by 103 out of the 185 patients (Table 5). The general level of satisfaction was high with $95 \%$ of the patients marking a score of seven out of 10 and $70 \%$ nine out of 10 to indicate how pleased they were

Table 5 The main results of the SPC satisfaction survey

\begin{tabular}{|c|c|c|c|}
\hline Question & Yes & No & Blank \\
\hline (1) Has SPC made a difference to your life? & $89 \%$ & $8 \%$ & $3 \%$ \\
\hline (2) Has SPC improved your life? & $82 \%$ & $6 \%$ & $12 \%$ \\
\hline (3) Has the SPC made your life more unsatisfactory? & $4 \%$ & $80 \%$ & $16 \%$ \\
\hline (4) On a scale of $0-10(0=$ worst. $10=$ best $)$ can you indicate & $95 \%>7 / 10$ & $5 \%<3 / 10$ & \\
\hline how pleased you are with your catheter? & $70 \%>9 / 10$ & & \\
\hline $\begin{array}{l}\text { (5) Would you have a catheter inserted again if this opportun- } \\
\text { ity were offered to you? }\end{array}$ & $69 \%$ & $14 \%$ & $17 \%$ \\
\hline $\begin{array}{l}\text { (6) Would you recommend this type of drainage to similarly } \\
\text { affected patients? }\end{array}$ & $79 \%$ & $7 \%$ & $16 \%$ \\
\hline
\end{tabular}


a

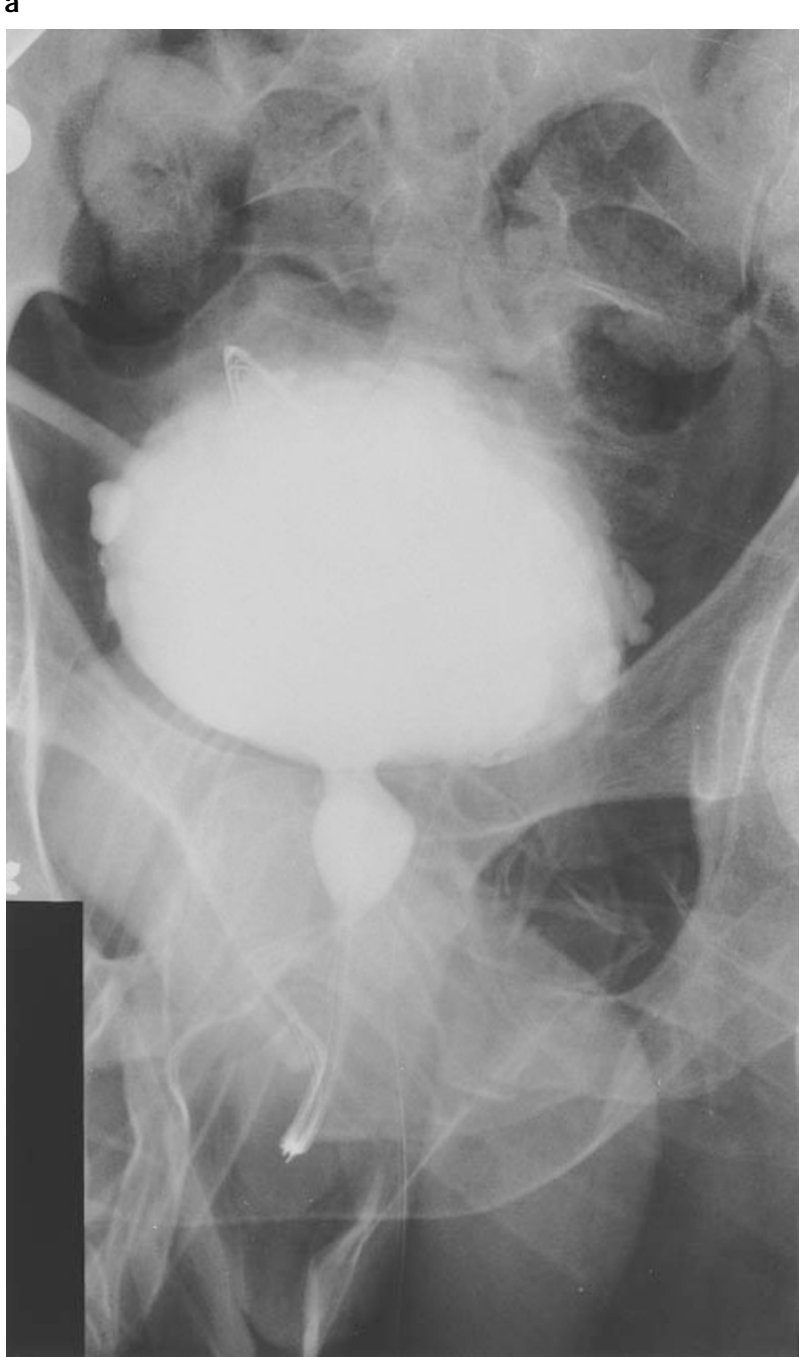

b

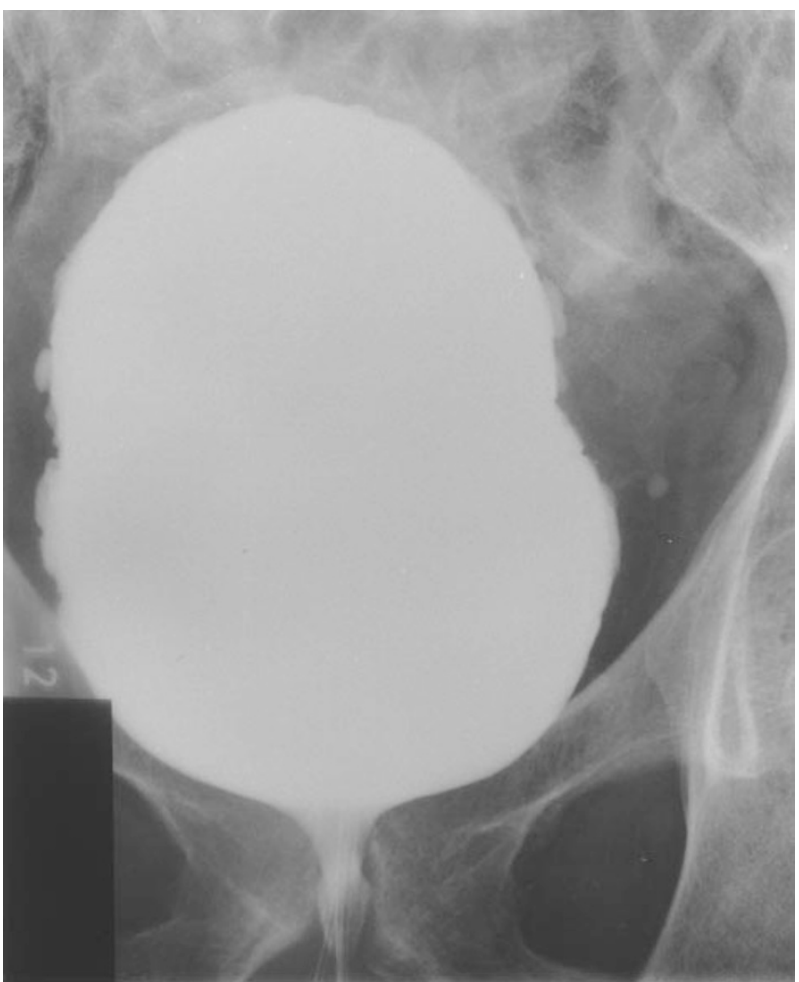

Figure 1 (a) Cystogram taken during Video-cystometry in a 28 year old male paraplegic patient practising CISC with adequate anti-cholinergic therapy. The bladder is grossly trabeculated, there is evidence of vesicoureteric reflux on the right, and the bladder neck is wide open. This patient has detrusor sphincter dyssynergia with bilateral hydronephorosis shown on upper tract ultrasound. (b) The same patient 5 months later showing clear improvement. The bladder morphology has improved, the reflux has disappeared and ultrasound examination is now normal

with SPC. Furthermore, $82 \%$ of the patients indicated that the SPC had positively improved the quality of their life and $79 \%$ had no hesitation in recommending this form of bladder management to similarly affected patients. However, between $3-17 \%$ of the patients omitted to answer the main questions of the survey.

\section{Discussion}

Clean intermittent self-catheterisation is currently the most popular method of bladder management following spinal cord injury. ${ }^{7}$ However, in a previous report from this unit $50 \%$ of the female patients discharged home on CISC regime failed to continue with this method. ${ }^{8}$ Persistent incontinence despite anti-cholinergic therapy was the main reason for the failure of the technique. In this study just fewer than $50 \%$ of the patients were females with a similar outcome. Suprapubic catheters have been used with success in the early management of traumatic spinal cord lesions ${ }^{9}$ and compared by prospective study with CISC for this use $^{10}$ with no reported difference between the two methods of management, although SPC was found to be a cheaper alternative.

However, Hackler ${ }^{5}$ condemned the long-term use of the SPC for causing as much renal damage in 5 years as urethral catheterisation caused in 20 years. His experience was based on 31 patients over a period of 8 years. But despite the fact that 24 patients out of the 31 had clinically significant detrusor hyper-reflexia, only six were on anti-cholinergic therapy. It is interesting to note that four out of these six patients 


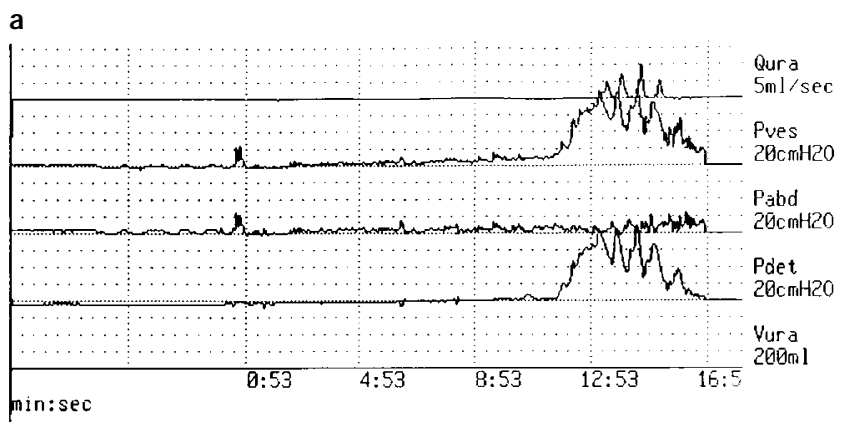

b

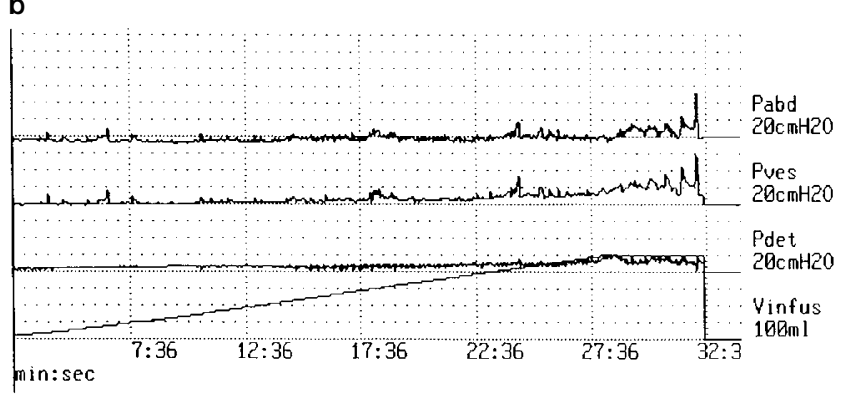

Figure 2 (a) Urodynamic trace of a 30 year old male tetraplegic patient demonstrating detrusor hyper-reflexia with peak pressures in the region of $120 \mathrm{~cm} \mathrm{H}_{2} \mathrm{O}$. (b) The same patient 6 months after the insertion of a suprapubic catheter showing clear urodynamic improvement with reduction in the maximal detrusor pressure from $120 \mathrm{~cm} \mathrm{H}_{2} \mathrm{O}$ to $15 \mathrm{H}_{2} \mathrm{O}$. The patient was on anti-cholinergic therapy for about a year before and 10 months after the catheterisation

maintained normal upper tracts. Furthermore, all patients in Hackler's series were on free bladder drainage.

This study demonstrates statistically significant reduction in the average maximum detrusor pressure following SPC in-patients with neuropathic bladder dysfunction. Furthermore, there is both structural and functional preservation of the renal tracts according to ultrasonography and serum creatinine levels. VCMG shows maintenance of cystometric bladder capacity. Subjectively there is $85 \%$ improvement in bladder morphology, although this finding must be interpreted with caution as mentioned earlier. The situation with regard to vesico-ureteric reflux is less clear especially in view of the small numbers involved.

It is encouraging to find that the policy of catheter clamping and anti-cholinergic therapy in presence of clinically significant detrusor hyper-reflexia has been followed by most of the patients. However, it is disappointing to discover that more catheters are not changed in the community, especially by the district nurse and the general practitioner. This places unnecessary strain on the hospital staff. More help should be sought from the community and to this end it is encouraging to find a reasonable level of participation by the patients and their carers.
It is disappointing to find that the $30 \%$ incidence of complaints with $48 \%$ associated clinically significant bladder calculi has not changed much from our previously published experience. ${ }^{6}$ Recurrent catheter blockage remains the commonest complaint with a higher incidence than that reported by Stower and his colleagues. ${ }^{11}$ Two major differences may account for this discrepancy. First, $88 \%$ of patients in our series were incontinent as a result of traumatic neuropathic bladder dysfunction whereas in Stower's series 66\% suffered from multiple sclerosis. Second, in the latter study size 18-20 Fr catheters were employed, whereas $16 \mathrm{Fr}$ catheters were inserted in over $90 \%$ of our patients. Larger catheters were used if urinary leakage secondary to recurrent blockage became severe. More recently, despite our emphasis on attention to volume and variability of daily fluid intake by these patients, ${ }^{12}$ there has been no noted significant difference. Blockage of urinary catheters is thought to involve the production of bacterial biofilms which consist of bacterial cells, glycocalyx and crystals. ${ }^{13,14}$ This is the subject of our current research.

Recurrent asymptomatic, but much less so symptomatic urinary tract infection also continues to be a problem with long-term SPC. Usually the infection responds to conservative measures and we agree with Stickler and Chawla that limiting the use of antibiotics to prevent the emergence of multi-drug resistance is necessary. ${ }^{15} \mathrm{We}$ avoid prophylaxis and only treat symptomatic cases.

Persistent urethral leakage was found to be less of a problem compared to our experience with CISC. ${ }^{8}$ It was found to be more common in females and associated with catheter blockage or prolonged clamping. The semi-predictable nature of urethral leakage of urine appears to have made the technique more acceptable to the patient compared to CISC. However, it is clear that those patients who regularly clamped catheters and were on adequate anticholinergic therapy for detrusor hyper-reflexia had the lowest incidence of complaints.

The most serious complication was a $2.7 \%$ incidence of small bowel injury, with one consequent fatality. It is educational to note that this occurred despite the fact that the operator involved was a senior surgeon with an enormous experience of this procedure. The technique has since been modified for the high-risk patient. We consider all obese patients with previous lower abdominal surgery and small contracted and fibrotic bladders which do not easily expand during cystoscopy to be at a high risk of sustaining small bowel injury. For such patients we recommend a small formal dissection to identify the bladder followed by a stab introduction of the 'ADDa-CATH' under direct vision, thus avoiding bowel injury.

Although the long-term consequences of SPC are unknown, there does appear to be an increased risk of squamous cell carcinoma in the region of $8 \%$ after 25 years of catheterisation. ${ }^{16}$ Although in our series we 
have not yet seen any neoplastic changes, this is clearly a dilemma and another compelling reason to minimise the use of long-term indwelling catheters. But, the fact remains that there is a high reported level of satisfaction and an increasing demand for this method of bladder management by patients. Our policy is to inform all patients of this potential risk and to exercise close vigilance with regular cystoscopies and random bladder biopsies after 5 years of catheterisation.

\section{Conclusions}

We consider SPC to be an effective and well tolerated method of management in selected patients with neuropathic bladder dysfunction for whom only major surgery would otherwise provide a solution to incontinence. It is encouraging to find maintained bladder volumes, reduced maximum detrusor pressures and preservation of upper tracts which we feel justify continuation of the policy of catheter clamping and anti-cholinergic therapy in patients with clinically significant detrusor hyper-reflexia. This procedure is not without hazard even in expert hands and therefore not to be lightly advocated. However, there is a high level of satisfaction and an increasing demand for this method of bladder management. In high-risk groups, we recommend SPC insertion under direct vision to prevent bowel injury. Finally, although we have not as yet encountered neoplastic changes in the bladder, this is another compelling reason to minimise the use of long-term SPC.

\section{Acknowledgements}

We would like to thank Helen Baywater for her assistance in retrieving the Urodynamic data.

\section{References}

1 Cook JB, Smith PH. Percutaneous suprapubic cystostomy after spinal cord injury. Br J Urol 1976; 48: 119-121.

2 Barkin M, Dolfin D, Herschorn S, Bharatwal N, Comisarow R. The urological care of the spinal cord injury patient. J Urol 1983; 129: $335-339$.

3 McMaster WC, Nicholas JJ, Rosen JS. Intermittent catheterisation for spinal-cord injury patients with chronic indwelling uretheral catheters. Arch Phy Med Rehab 1972; 53: 563-567.

4 Feneley RCL. The management of female incontinence by suprapubic catheterisation with or without urethral closure. $\mathrm{Br}$ J Urol 1983; 55: 203-207.

5 Hackler RH. Long-term suprapubic cystostomy drainage in spinal cord injury patients. Br J Urol 1982; 54: 120-121.

6 Barnes DG, Shaw PJR, Timoney AG, Tsokos N. Management of the neuropathic bladder by suprapubic catheter. Br J Urol 1993; 72: $169-172$

7 Stover SL. 3rd Annual Report National Spinal Cord Injury Statistical Centre, University of Alabama, Birmingham, AL, 1985.

8 Timoney AG, Shaw PJR. Urological outcome in female patients with spinal cord injury: The effectiveness of intermittent catheterisation. Paraplegia 1990; 28: 556-563.

9 Peatfied RC, Burt AA, Smith PH. Suprapubic catheterisation after spinal cord injury. A follow up report. Paraplegia 1983; 21: $220-226$.

10 Grundy DJ, Fellows GI, Gillett AP et al. A comparison of finebore suprapubic and intermittent urethral catheterisation regime after spinal cord injury. Paraplegia 1983; 21: 227-232.

11 Stower MJ, Massey JA, Feneley RCL. Urethral closure in management of urinary incontinence. Urology 1989; 34: $246-$ 248 .

12 Burr RG, Nuseibeh I. The blocking urinary catheter: the role of variation in urine flow. Br J. Urol 1995; 76: $61-65$.

13 Ramsay JWA, Garnham AJ, Mulhall AB et al. Biofilms, bacteria and bladder catheters. A clinical study. Br J Urol 1989; 64: $395-$ 398.

14 Hedelin H, Bratt C-G, Eckerdal G, Lincoln K. Relationship between urease-producing bacteria, urinary $\mathrm{pH}$ and encrustation on indwelling urinary catheters. Br J Urol 1991; 67: 527-531.

15 Stickler DJ, Chawla JC. An appraisal of antibiotic policies for urinary tract infections in patients with spinal cord injuries undergoing long-term catheterisation. Paraplegia 1988; 26: 215 225.

16 Locke JR, Hill DE, Walzer Y. Incidence of squamous cell carcinoma in patients with long-term catheter drainage. $J$ Urol 1985; 133: $1034-1035$. 